\title{
The nonlinear relation between perceived employability and emotional exhaustion
}

\section{Ellen Peeters}

Nele De Cuyper

Hans De Witte

Supported by a grant from the KU Leuven, OT/11/010 Research Group Work, Organizational and Personnel Psychology 


\section{Introduction (1/4)}

Perceived employability:

'The perceived likelihood of obtaining alternative employment (...) on the internal or external labor market' (De Cuyper \& De Witte, 2010).

- Perceived internal employability

- Perceived external employability

\section{Emotional exhaustion:}

Feeling of lack of energy due to 'being over-extended and depleted of one's emotional resources'

(Maslach, Schaufeli, \& Leiter, 2001, p. 399) 


\section{Introduction (2/4)}

\section{Perceived employability}

- Resource that promotes well-being and reduces

ill-being (Berntson \& Marklund, 2007)

- But: low correlations between perceived employability and emotional exhaustion

- Is it that simple or is it more complex?

Linear versus curvilinear relations (e.g. Dunford, Shipp, Boss, Angermeier, \& Boss, 2012)
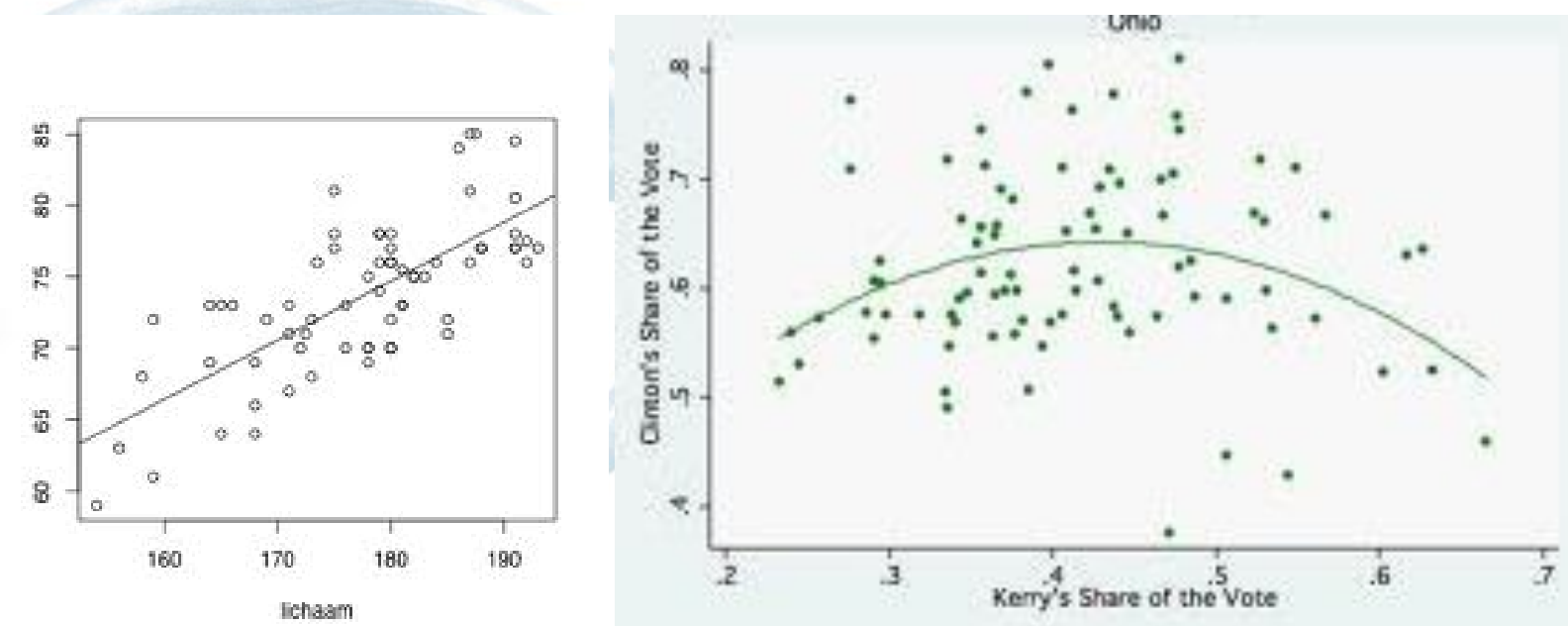


\section{Introduction (3/4)}

High perceived internal employability

- Social exchange theory (e.g. Chambel \& Sobral, 2011)

- Reciprocity (De Cuyper \& De Witte, 2011) 


\section{Introduction (4/4)}

Very low perceived external employability

- Locked-in one's profession (Aronsson \& Göransson, 1999)

- Reciprocity

Very high perceived external employability

- Social exchange theory (e.g. Emerson, 1976)

- Decision stress 


\section{Aim of this research:}

(1) Test nonlinear relations between perceived employability and emotional exhaustion

(2) Compare perceived internal employability with perceived external employability in relation to emotional exhaustion 


\section{Hypotheses}

- H1: Perceived internal employability relates negatively to emotional exhaustion (linear relationship)

- H2: Perceived external employability relates quadratic emotional exhaustion (curvilinear relationship) 


\section{Method (1/3)}

\section{Sample: 334 Participants}

- Age:

- Range: 20-61y

- Average: 40y

\begin{tabular}{|l|l|l|}
\hline Gender & N & $\%$ \\
\hline Male & 160 & $48 \%$ \\
\hline Female & 174 & $52 \%$ \\
\hline Education level & $\mathrm{N}$ & $\%$ \\
\hline Lower education & 176 & $53 \%$ \\
\hline Higher education & 158 & $47 \%$ \\
\hline Job level & $\mathrm{N}$ & $\%$ \\
\hline Blue-collar & 112 & $34 \%$ \\
\hline White-collar & 222 & $66 \%$ \\
\hline
\end{tabular}




\section{Method (2/3)}

Measures

- Perceived employability (De Cuyper \& De Witte, 2008)

- Perceived internal employability $(\alpha=.94)$

- Perceived external employability $(\alpha=.93)$

- Scale: 1-5

- Internal: 8 items: e.g. "I am optimistic that I would find another job with this employer, if I looked for one"

- External: 8 items: e.g. "I am optimistic that I would find another job elsewhere, if I looked for one"

- Burn-out (Dutch version (Schaufeli \& Van Dierendonck, 2000) of the Maslach Burnout Inventory, Maslach \& Jackson, 1981): 5 items

- Emotional exhaustion $(\alpha=.91)$

- Scale: 1-7

- "I feel burned out from work". 


\section{Method (3/3)}

- Curve estimations

- Based on all raw results

- Aim: consider several types of relations (e.g. exponential, quadratic, linear)

$-R^{2}$

- Plots 


\section{Results}

- Perceived internal employability

- Linear: $\beta=-.14, p=.01$

- Quadratic: $\beta=.22, p=.41$

- Perceived external employability

- Linear: $\beta=-.03, p=.65$

- Quadratic: $\beta=.60, p=.03$ 


\section{Results}

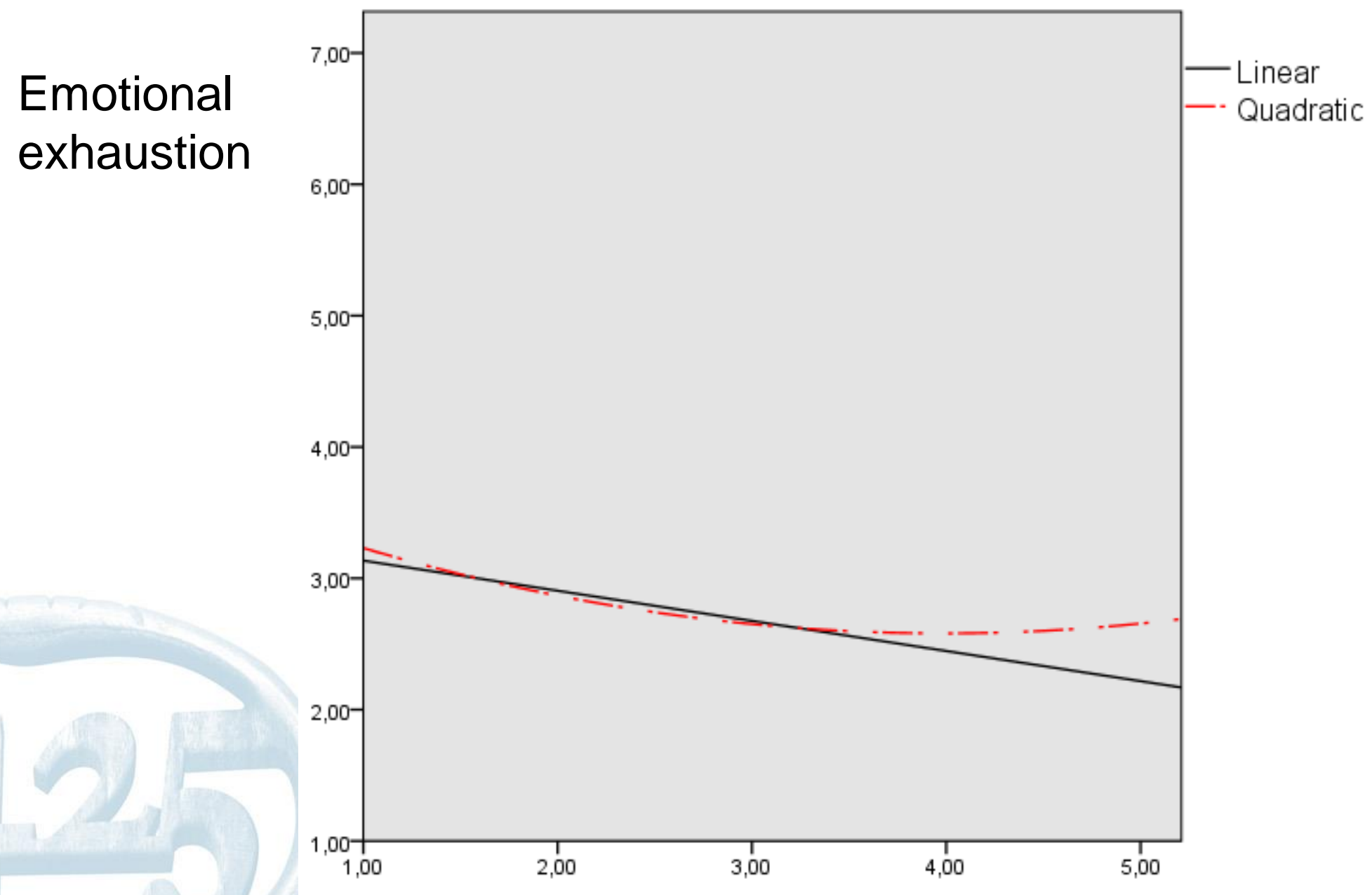

Perceived internal employability 


\section{Results}

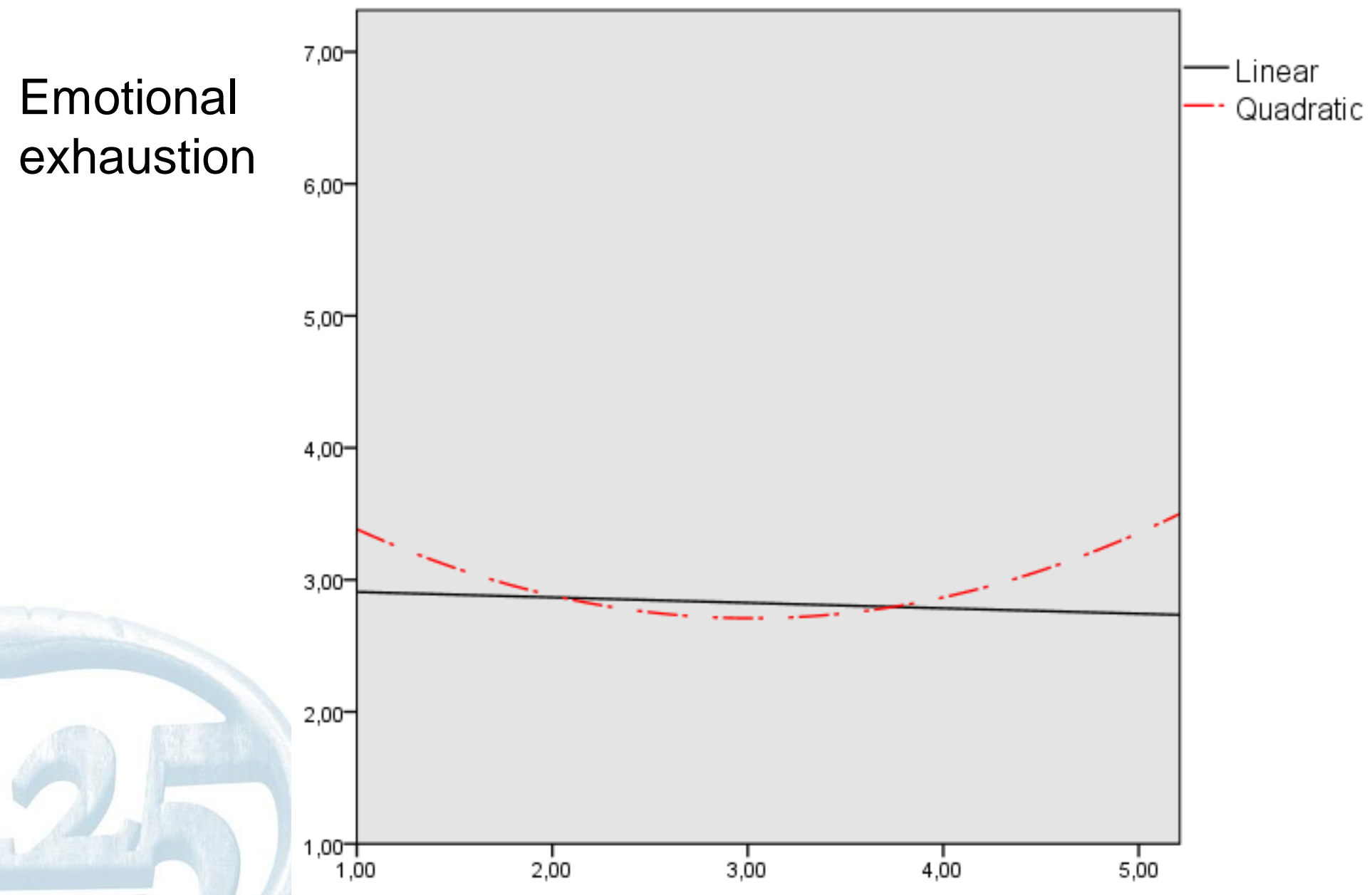

Perceived external employability 


\section{Discussion (1/2)}

Conclusion:

- Higher perceived internal employability relates to lower emotional exhaustion

- Too high and too low perceived external employability relates to higher emotional exhaustion.

Limitation:

- Cross-sectional 


\section{Discussion (2/2)}

Take home message:

- Downside of perceived employability

- Linear assumption can be false

- Importance of distinguishing between internal and external employability 


\section{References}

- Aronsson, G., \& Göransson, S. (1999). Permanent employment but not in a preferred occupation: psychological and medical aspects, research implications. Journal of Occupational Health Psychology, 4, 152-163.

- Berntson, E., \& Marklund, S. (2007). The relationship between perceived employability and subsequent health, Work \& Stress, 21(3), 279-292.

- De Cuyper, N., \& De Witte, H. (2008). Gepercipieerde kans op een baan versus een betere baan: Relaties met arbeidstevredenheid en welzijn. Gedrag \& Organisatie, 21, 475-492.

- Dunford, B. B., Shipp, A. J., Boss, R. W., Angermeier, I., Boss, A. D. (2012). Is Burnout Static or Dynamic? A Career Transition Perspective of Employee Burnout Trajectories. Journal of Applied Psychology, Advance online publication. doi: $10.1037 / \mathrm{a} 0027060$.

- Lazarus, R. S., \& Folkman, S. (1984). Stress, appraisal and coping. New York: Springer Publishing Company.

- Maslach, C., Schaufeli, W. B., \& Leiter, M. P. (2001). Job burnout. Annual Review of Psychology, 52, 397-422.

- Schaufeli, W. B., \& Van Dierendonck, D. (2000). Maslach burnout inventory: Nederlandse versie [Maslach burnout inventory: Dutch version]. Lisse [The Netherlands]: Swets Zeitlinger. 
Thank you for your attention!

Any questions?

Ellen.Peeters@ppw.kuleuven.be 


\section{Correlation matrix}

\begin{tabular}{|c|c|c|c|c|c|c|c|c|}
\hline \multicolumn{9}{|c|}{ Correlations } \\
\hline & & GENDER & AGE & DIPLOWA & ORG-LEVEL & PE-I & PE-E & $E-E$ \\
\hline \multirow[t]{3}{*}{$\overline{\text { GENDER }}$} & Pearson Correlation & 1 & .012 &,- 002 & .067 & $172^{x \times}$ & .096 &,- 025 \\
\hline & Sig. (2-tailed) & &, 831 &, 973 & .245 &, 002 &, 086 & .661 \\
\hline & $\mathbb{N}$ & 336 & 336 & 336 & 302 & 320 & 320 & 320 \\
\hline \multirow[t]{3}{*}{$\mathrm{AGE}$} & Pearson Correlation &, 012 & 1 & $-.226^{x \pi}$ &,- 060 &,$- 174^{x \pi}$ &,$- 386^{x x}$ & .047 \\
\hline & Sig. (2-tailed) &, 831 & & 000 &, 300 &, 002 &, 000 & .401 \\
\hline & $\mathbb{N}$ & 336 & 336 & 336 & 302 & 320 & 320 & 320 \\
\hline \multirow[t]{3}{*}{ DIPLOMA } & Pearson Correlation &,- 002 &,$- 226^{x \pi}$ & 1 & $.682^{x \pi}$ & $.130^{x}$ &, 086 &,- 060 \\
\hline & Sig. (2-tailed) & 973 &, 000 & & 000 &, 020 &, 123 & .282 \\
\hline & $\mathbb{N}$ & 336 & 336 & 336 & 302 & 320 & 320 & 320 \\
\hline \multirow[t]{3}{*}{ ORG-LEVEL } & Pearson Correlation & .067 &,- 060 & $.682^{x x}$ & 1 & $.199^{x \pi}$ &, 008 &,- 015 \\
\hline & Sig. (2-tailed) &, 245 &, 300 & 000 & & , 001 &, 891 & .803 \\
\hline & $N$ & 302 & 302 & 302 & 302 & 290 & 290 & 290 \\
\hline \multirow[t]{3}{*}{ PE-I } & Pearson Correlation & $.172^{x \pi}$ &,$- 174^{x \pi}$ & $.130^{x}$ & $.199^{x \pi}$ & 1 & $.239^{x \pi}$ &,$- 132^{x}$ \\
\hline & Sig. (2-tailed) &, 002 &, 002 &, 020 & 001 & &, 000 & ,018 \\
\hline & $\mathbb{N}$ & 320 & 320 & 320 & 290 & 320 & 320 & 320 \\
\hline \multirow[t]{3}{*}{ PE-E } & Pearson Correlation & .096 &,$- 386^{x x}$ & ,086 & ,008 & $.239^{x \pi}$ & 1 &,- 023 \\
\hline & Sig. (2-tailed) & ,086 & ,000 &, 123 &, 891 & , 000 & & .679 \\
\hline & $N$ & 320 & 320 & 320 & 290 & 320 & 320 & 320 \\
\hline \multirow[t]{3}{*}{$\mathrm{E}-\mathrm{E}$} & Pearson Correlation &,- 025 &, 047 &,- 060 &,- 015 &,$- 132^{x}$ &,- 023 & 1 \\
\hline & Sig. (2-tailed) & .661 & .401 & .282 & .803 &, 018 & .679 & \\
\hline & $\mathbb{N}$ & 320 & 320 & 320 & 290 & 320 & 320 & 320 \\
\hline \multicolumn{8}{|c|}{$\begin{array}{l}* \text { Correlation is significant at the } 0.01 \text { level (2-tailed). } \\
* \text { Correlation is significant at the } 0.05 \text { level (2-tailed). }\end{array}$} & 18 \\
\hline
\end{tabular}

